\title{
SiPhotonics/GaAs 28-GHz Transceiver for mmWave-over-Fiber Laser-Less Active Antenna Units
}

\author{
L. Bogaert ${ }^{1,2, *}$, J. Van Kerrebrouck ${ }^{1}$, H. Li ${ }^{1}$, I. L. de Paula ${ }^{1}$, K. Van Gasse ${ }^{2}$, S. Lemey ${ }^{1}$, \\ H. Rogier1, P. Demeester ${ }^{1}$, G. Roelkens ${ }^{2}$, J. Bauwelinck ${ }^{1}$, G. Torfs ${ }^{1}$ \\ ${ }^{1}$ IDLab, INTEC, Ghent University - imec, 9052 Ghent, Belgium \\ ${ }^{2}$ Photonics Research Group, INTEC, Ghent University - imec, 9052 Ghent, Belgium \\ *laurens.bogaert@ugent.be
}

\begin{abstract}
We demonstrate a $28 \mathrm{GHz}$ radio-over-fiber system with laser-less, low-cost active antenna units using silicon photonics and a GaAs driver and LNA. 7-Gb/s downlink and uplink throughput was achieved over $2 \mathrm{~km}$ SSMF and $5 \mathrm{~m}$ wireless.

OCIS codes: (060.2330) Fiber optics communications, (060.5625) Radio frequency photonics
\end{abstract}

\section{Introduction}

Meeting the demands for future wireless mobile communication will require significant changes in the underlying network [1]. A first important change is the migration to higher carrier frequencies as these bands offer more bandwidth and are less congested than the sub-6 GHz bands. Secondly, a small-cell approach will be adopted to increase the overall data capacity of the network. To allow for the densification of the network, a centralized approach with distributed low-complexity active antenna units (AAUs) is of paramount importance. In such a configuration, centralized offices (COs) contain the high-complexity functionalities, such as the generation and processing of the RF signal, and subsequently distribute the generated data to the intended AAU using radio-overfiber (RoF) technology. Typical RoF implementations for mmWave distribution rely on IF-over-Fiber and accomplish the frequency up-conversion at the AAU $[2,3]$. This approach requires the distribution of a synchronous carrier which is used to generate a local oscillator signal in the AAU.

In this work, the complexity of the AAU is further reduced by adopting RF-over-Fiber (RFoF). Furthermore, a reflective electro absorption modulator (EAM), with compact footprint, is used to realize laser-free AAUs, thereby further reducing cost, complexity and weight. In contrast to the broadband approaches used in prior works, a dedicated EAM-driver and photoreceiver are designed for optimal performance in the $28 \mathrm{GHz}$ band using a combination of GaAs pHEMT electronics and silicon photonics. The signal processing and computing resource allocation are transferred to the $\mathrm{CO}$ to further simplify the AAU and reduce the latency. This proposed RFoF system features low-complexity, low-cost and easy to install AAUs, which is highly desired in centralized networks and distributed antenna systems (DAS). Besides small signal characterization, the performance and throughput of the $\mathrm{RFoF}$ system is evaluated for mmWave communications demonstrating $12 \mathrm{~Gb} / \mathrm{s}$ transmission over $2 \mathrm{~km}$ standard single mode fiber (SSMF). After introduction of a $5 \mathrm{~m}$ wireless path $7 \mathrm{~Gb} / \mathrm{s}$ transmission is obtained.

\section{Experimental setup}

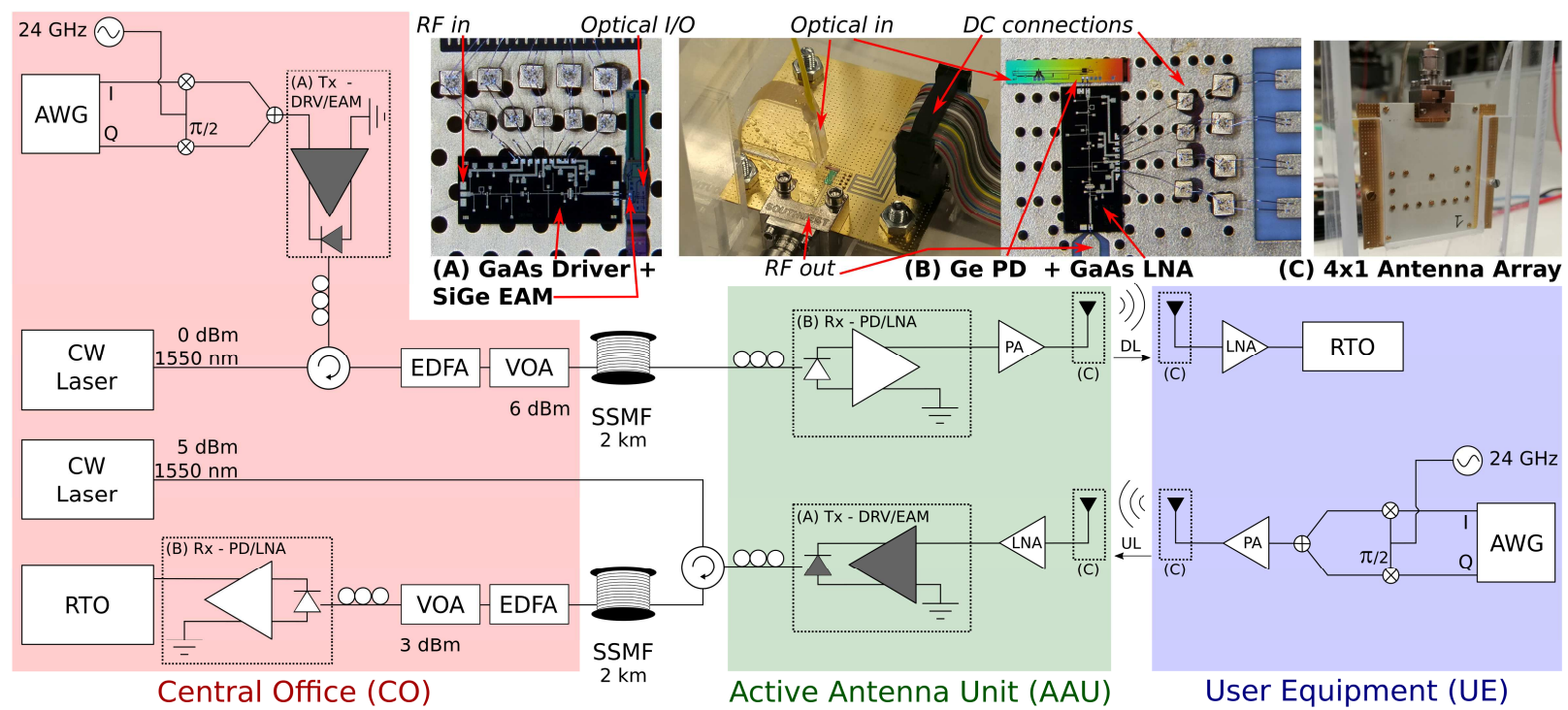

Fig. 1: Block diagram and experimental setup for bidirectional $28 \mathrm{GHz}$ RFoF link 
The experimental setup, consisting of both the uplink and downlink of the proposed RFoF system, is shown in Fig. 1. In this work, the 5G New Radio channels nr257/258 were targeted with frequency ranges between 24.25 and $29.5 \mathrm{GHz}$. Furthermore, nr257/258 adopt a time division duplexing (TDD) scheme [4].

The downlink path starts with an arbitrary waveform generator (AWG) that generates an IF signal which is subsequently up-converted to the RF frequency. The generated RF signal is amplified by a dedicated narrowband GaAs EAM-driver, which offers a small signal gain of $25.2 \mathrm{~dB}$ over a 3-dB bandwidth between 24.4 and $29.5 \mathrm{GHz}$ with a noise figure of $2.0 \mathrm{~dB}$. The driver has an input referred 1-dB compression point of $-20 \mathrm{dBm}$ and consumes $124 \mathrm{~mW}$. The output of the GaAs driver is fed to a SiGe reflective EAM coupled to silicon waveguides and modulates the incident continuous wave $(\mathrm{CW}) 1550 \mathrm{~nm}$ laser tone incident on the EAM. Since the modulator is reflective, an optical circulator is required to separate the modulated from the unmodulated light. The reflective EAM has a very compact footprint of $340 \mu \mathrm{m}$ by $220 \mu \mathrm{m}$ and is fabricated on the iSiPP50G silicon photonics platform with a bandwidth far beyond $28 \mathrm{GHz}$, which opens the opportunity to realize RFoF systems at even higher frequency bands, such as the extended frequency range in $5 \mathrm{G}$ New Radio and the $60-\mathrm{GHz}$ band used by WiGig.

An erbium doped fiber amplifier (EDFA) and a variable optical attenuator (VOA) are used to set the power launched into the SSMF. At the AAU, the photoreceiver converts the light back to the RF domain and subsequently amplifies the signal. The devised photoreceiver comprises a silicon waveguide coupled Ge-on-Si photodetector (PD) and a co-designed GaAs low noise amplifier (LNA). The LNA offers $24 \mathrm{~dB}$ gain, corresponding to $224 \mathrm{~V} / \mathrm{W}$ external conversion gain, over a 3-dB bandwidth between 23.5 and $31.5 \mathrm{GHz}$ [5]. Its associated noise figure is 2.1 $\mathrm{dB}$ and an output referred third order intercept point up to $26.5 \mathrm{dBm}$ can be obtained with a power consumption of $303 \mathrm{~mW}$. The devised narrowband $\mathrm{GaAs} / \mathrm{SiGe}$ transceiver has a total power consumption of $427 \mathrm{~mW}$ (driver and receiver). A commercial power amplifier (HMC943) is added to ensure that the signal fed to the antenna is sufficiently strong (approximately $10 \mathrm{dBm}$ ). Furthermore, $4 \times 1$ linear and passive antenna arrays with integrated Wilkinson splitters are used to achieve beamforming gain in the broadside direction. The downlink signal received by the antenna at the user equipment (UE) is first amplified and subsequently monitored by a real-time oscilloscope (RTO, Keysight DSA-Z634A). The captured data was demodulated offline in Matlab.

The uplink path first generates an RF signal and subsequently passes the signal over the wireless link. Next, the signal is amplified with a commercial low noise amplifier (HMC1040) and fed to the EAM-driver which modulates the incident $\mathrm{CW}$ laser tone. A reflective EAM was used to enable laser-free operation of the AAU. To separate the $\mathrm{CW}$ tone incident on the reflective EAM from the modulated light coming from the EAM, an optical circulator is used. Subsequently, the light passes through SSMF and is converted back to the electrical domain at the central office by making use of the aforementioned photoreceiver.

\section{Results and Discussion}

The transfer function of the RFoF link in optical back-to-back (OB2B) starting from the input of the EAM-driver to the output of the photoreceiver is shown in Fig. 2. The 3-dB bandwidth of the link spans from 24.7 to $28.6 \mathrm{GHz}$ and shows a small signal gain of $28.4 \mathrm{~dB}$ when $3 \mathrm{dBm}$ optical power is incident on the photoreceiver.

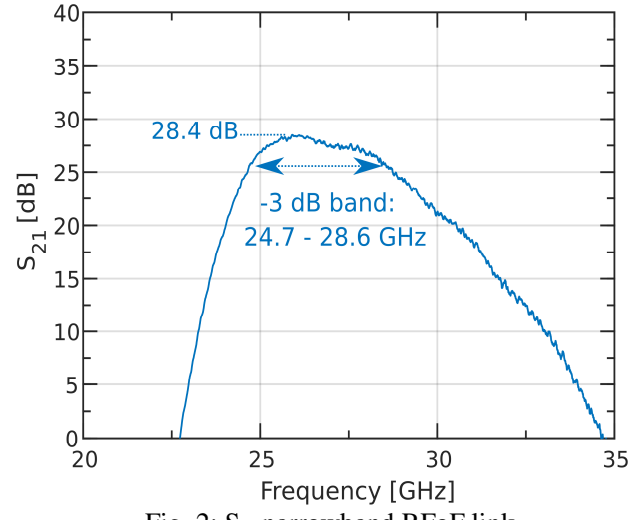

Fig. 2: $\mathrm{S}_{21}$ narrowband RFoF link

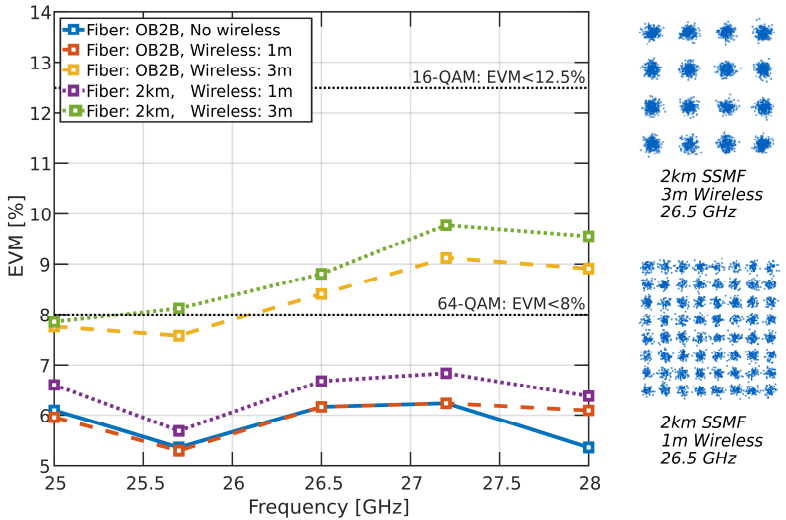

Fig. 3: Downlink single carrier - 5 x 400 MBaud Multiband, exploring maximum RFoF link capacity.

To explore the maximum RFoF link capacity, downlink multiband single carrier experiments were performed. Five $400 \mathrm{MBd}$ channels centered at 25.0, 25.7, 26.5, 27.2 and 28.0 GHz were transmitted simultaneously over the fiber-wireless link. The EVM values (normalized to the average power) of the transmitted data are measured in the absence of a wireless channel for an OB2B link and compared to different wireless scenarios in Fig. 3. For $1 \mathrm{~m}$ wireless, the EVM stays well below the 8\% requirement for 64-QAM [6]. It should be pointed out that the optical insertion loss of $2 \mathrm{~km}$ SSMF has a limited impact on the signal reception quality. When the wireless distance is 
increased to $3 \mathrm{~m}$, the $12.5 \%$ EVM requirement for 16-QAM is still met [6]. Consequently, using 5-channel multiband single carrier data transmission allows for data rates up to $12 \mathrm{~Gb} / \mathrm{s}$ over $1 \mathrm{~m}$ wireless distance and up to $8 \mathrm{~Gb} / \mathrm{s}$ over $3 \mathrm{~m}$ wireless distance in a typical indoor environment. At larger distances, fading significantly degrades the signal quality.

To overcome equalization challenges after fading, orthogonal frequency division multiplexing (OFDM) signals were also evaluated for this RFoF system. OFDM signals make the data transmission over the wireless channel more robust at the cost of increased requirements on the dynamic range of the $\mathrm{E} / \mathrm{O}$ and $\mathrm{O} / \mathrm{E}$ converters and its associated drivers and amplifiers [7]. The OFDM signal parameters used for each channel and its data rate are summarized in Fig. 4(a). Each OFDM channel can support $2.34 \mathrm{~Gb} / \mathrm{s}$ using 16-QAM. The uplink and downlink path are tested separately due to the envisioned TDD duplexing mode [4]. For one OFDM channel, the EVM after $2 \mathrm{~km}$ fiber was below 4\%. For three OFDM channels after 2km fiber, all EVMs were below $8 \%$ [6] and the averaged EVM was around 6\%, as shown in Fig. 4(b) and 4(c). For 1m wireless distance, the measured EVMs can even support 64QAM. An aggregated capacity of $7.02 \mathrm{~Gb} / \mathrm{s}$ was achieved over $2 \mathrm{~km}$ SSMF and $5 \mathrm{~m}$ wireless distance for both downlink and uplink with an EVM that meets the 3GPP specification.

\begin{tabular}{|l|c|}
\hline Bandwidth & $800 \mathrm{MHz}$ \\
\hline $\begin{array}{l}\text { Number of } \\
\text { subcarriers }\end{array}$ & 512 \\
\hline $\begin{array}{l}\text { Number of } \\
\text { pilots }\end{array}$ & 16 \\
\hline $\begin{array}{l}\text { Number of DC, } \\
\text { null subcarriers }\end{array}$ & 28 \\
\hline $\begin{array}{l}\text { Cyclic prefix } \\
\text { (CP) size }\end{array}$ & $1 / 4$ \\
\hline $\begin{array}{l}\text { Peak-to- } \\
\text { average-ratio }\end{array}$ & $10.8 \mathrm{~dB}$ \\
\hline $\begin{array}{l}\text { Data rate per } \\
\text { channel (16- } \\
\text { QAM) }\end{array}$ & $2.34 \mathrm{~Gb} / \mathrm{s}$ \\
\hline
\end{tabular}

(a)

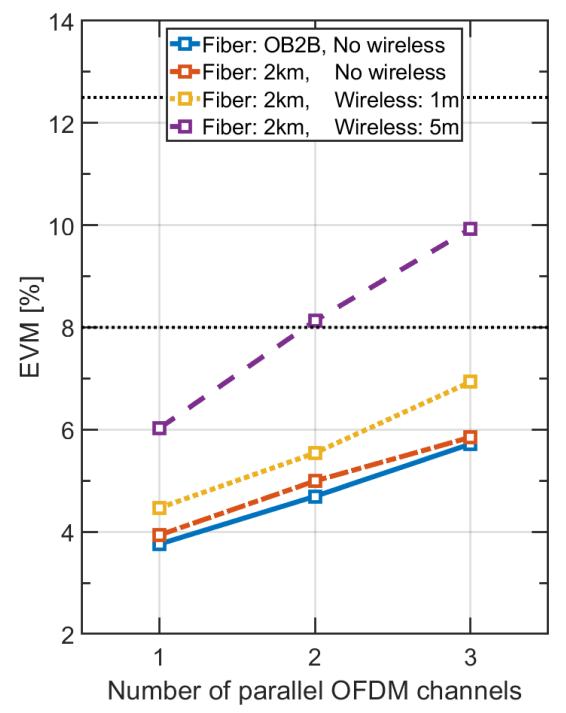

(b)

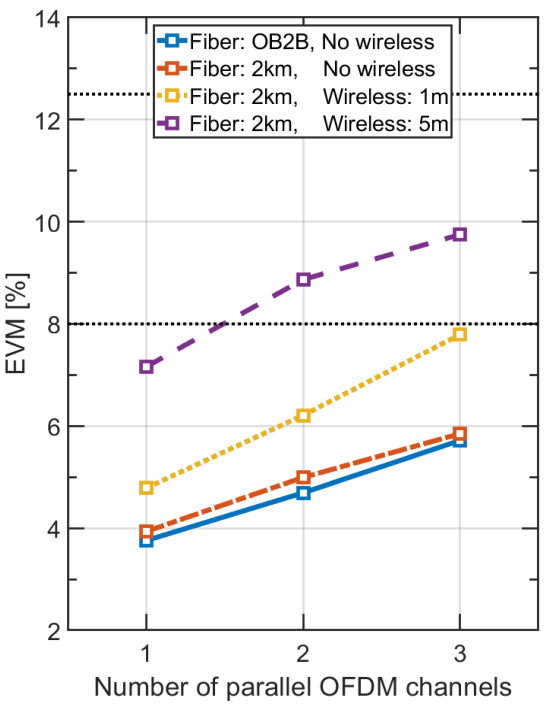

(c)

Fig. 4: (a) OFDM signal parameters. (b) Measured EVM in RFoF-wireless downlink. (c) Measured EVM in RFoF-wireless uplink.

\section{Conclusion}

We have demonstrated a very low complexity narrowband GaAs electronics/Si photonics transceiver for scalable $\mathrm{RFoF}$ architectures. The chipset consumes $427 \mathrm{~mW}$, introduces a link gain of $28.4 \mathrm{~dB}$ - with $3 \mathrm{dBm}$ optical power and supports a link bandwidth from 24.7 to $28.6 \mathrm{GHz}$. Furthermore, laser-free active antenna unit operation is enabled due to the reflective EAM used in the RFoF transmitters, which reduces the complexity of the active antenna units even further. With this transceiver, $12 \mathrm{~Gb} / \mathrm{s}$ over $2 \mathrm{~km} \mathrm{SSMF}$ was demonstrated and over $7 \mathrm{~Gb} / \mathrm{s} \mathrm{down-}$ and uplink were demonstrated for a $2 \mathrm{~km}$ fiber, $5 \mathrm{~m}$ wireless mmWave link with an EVM around $10 \%$.

\section{Acknowledgements}

Ghent University (BOF14/GOA/034), ERC Grant ATTO (695495), Methusalem funding, AFOSR (FA95501810015), H2020 5G-PHOS (761989).

\section{References}

[1] J. G. Andrews et al., "What Will 5G Be?" IEEE J. Sel. Areas Commun., 32 (6), 1065-1082, June 2014.

[2] J. Kim et al., "OTA enabled $147.4 \mathrm{~Gb} / \mathrm{s}$ eCPRI-equivalent-rate radio-over-fiber link cooperating with mmWave-based Korea Telecom $5 \mathrm{G}$ mobile network for distributed antenna system," OFC, 2019.

[3] N. Argyris et al., "A 5G mmWave Fiber-Wireless IFoF Analog Mobile Fronthaul Link With up to 24-Gb/s Multiband Wireless Capacity," J. Light. Technol., 37 (12), 2883-2891, June 2019.

[4] 3GPP, "TR 38.815: New Frequency Range for NR (24.25 - 29.5 GHz) - Release 15,” 2018.

[5] L. Bogaert et al., " 36 Gb/s Narrowband Photoreceiver for mmWave Analog Radio-over-Fiber," J. Light. Technol., 2020 [Early Access].

[6] 3GPP, "TR 36.104: LTE; Evolved Universal Terrestrial Radio Access (EUTRA); Base Station (BS) radio transmission and reception Release 15, v. 15.3.0,” 2018.

[7] A. Zaidi et al., "Waveform and Numerology to Support 5G Services and Requirements," IEEE Commun. Mag., 54 (11), 90-98, Nov. 2016. 